\title{
On the average minimum size of a manipulating coalition
}

\author{
Geoffrey Pritchard ${ }^{1}$, Arkadii Slinko ${ }^{2 \star \star \star}$ \\ 1 Department of Statistics, University of Auckland, Auckland, New Zealand, \\ e-mail: g.pritchardeauckland.ac.nz \\ 2 Department of Mathematics, University of Auckland, Auckland, New Zealand, e-mail: \\ a.slinkodauckland.ac.nz
}

Received: date / Revised version: date

Summary: We study the asymptotic average minimum manipulating coalition size as a characteristic of quality of a voting rule and show its serious drawback. We suggest using the asymptotic average threshold coalition size instead. We prove that, in large electorates, the asymptotic average threshold coalition size is maximised among all scoring rules by the Borda rule when the number $m$ of alternatives is 3 or 4 , and by $\lfloor m / 2\rfloor$-approval voting when $m \geq 5$.

Key words: Borda rule, approval voting, coalition, manipulability

\section{JEL Classification Numbers: D71}

\section{Introduction}

In 1973-75 Gibbard and Satterthwaite published a fundamental impossibility theorem which states that every non-dictatorial social choice function, whose range contains at least three alternatives, at certain profiles can be manipulated by a single individual [12,22]. After that, the natural question arose: if there are no perfect rules, which ones are the best, i.e. least manipulable? The answer to this question cannot be given in absolute terms. It stipulates introducing a certain index of manipulability of the rule and a certain model for the population. The answer may depend both on the choice of the index and on the choice of the model.

To answer this question, various indices of manipulability of voting rules have been introduced and studied $[1,8,14,20,21]$ and various models introduced [2,

\footnotetext{
* Arkadii Slinko is grateful to Persi Diaconis for suggesting the strategy of calculating order statistics for the case of dependent variables adopted in this article. He also thanks Steven Brams for bringing the article [6] to his attention.

${ }^{\star \star}$ Correspondence to: A.Slinko
} 
21]. Among the models the following two have gained the lion's share of attention. The first one, IC (Impartial Cuture) conjecture, assumes that all voters are independent. The second one, the IAC (Impartial Anonymous Culture) conjecture, assumes some degree of dependency.

Among the indices, the probability of obtaining a manipulable profile either for an individual or for a coalition has attracted most attention, both for the IC and for the IAC conjectures $[8,17,20]$.

In some cases, these indices have been calculated exactly, mainly in the threealternative case [11,17-19]. In others, they have been estimated in computer simulations $[1,8,14,20,24]$.

The probability of manipulation has been especially well-studied for the important class of scoring voting rules, and significant progress has been made in comparing them. In his seminal paper [21], Saari showed that in his "geometric" model, Borda is the least manipulable for the three-alternative case in relation to micro manipulation, but this does not extend to the case of four alternatives. Kim and Roush [15], on the other hand, proved that, asymptotically, when the number of voters tends to infinity Borda becomes coalitionally manipulable with probability 1.

Some other characteristics have also been used, mostly in computer simulations $[1,8,20]$. In this paper, we originally aimed to compare scoring rules under the IC, using the average minimum size of the coalition capable of manipulation as the principal characteristic of manipulability of the rule. The greater this characteristic, the better the rule. This characteristic was first introduced by Chamberlin [8], where he estimated it for four different rules by means of computer simulation.

To our surprise we discovered that while Chamberlin's characteristic is meaningful for most classical rules, it is biased towards antiplurality, and the closer the rule gets to the antiplurality, the larger is its average minimum coalition size. Hence, there are no optimal rules in relation to this characteristic at all. We suggest using the asymptotic average threshold coalition size instead. This new characteristic coincides with the average minimum coalition size on classical rules but does not have the bias towards antiplurality. We show how to calculate the limiting value of this characteristic when $n \rightarrow \infty$, and discuss which rule is optimal for large electorates. We prove that, among all scoring rules, Borda is the optimal rule in this sense in three- and four-alternative elections. When the number of alternatives $m$ is five or more, $\lfloor m / 2\rfloor$-approval voting is optimal, where for each voter most preferred $\lfloor m / 2\rfloor$ alternatives are considered approved.

\section{Scoring Rules}

Let $A$ and $N$ be two finite sets of cardinality $m$ and $n$ respectively. The elements of $A=\left\{a_{1}, \ldots, a_{m}\right\}$ will be called alternatives, the elements of $N$ agents. We will denote agents as $1,2, \ldots, n$. We assume that the agents have preferences over the set of alternatives. By $\mathcal{L}=\mathcal{L}(A)$ we denote the set of all linear orders on $A$; they represent the preferences of agents over $A$. The elements of the Cartesian product

$$
\mathcal{L}^{n}=\mathcal{L} \times \ldots \times \mathcal{L} \quad(n \text { times })
$$


are called profiles. They represent the collection of preferences of the society $N$. If a linear order $R_{i} \in \mathcal{L}$ represents the preferences of the $i$-th agent, then by $a R_{i} b$, where $a, b \in A$, we denote that this agent prefers $a$ to $b$. A family of mappings $F_{n}: \mathcal{L}^{n} \rightarrow A, n \in \mathbb{N}$, is called a social choice function (SCF).

Definition 1 Let $u=\left(R_{1}, \ldots, R_{n}\right)$ be a profile. We say that a profile u' occurred as a result of misrepresentation by agents of a coalition $C=\left\{i_{1}, \ldots, i_{k}\right\} \subseteq N$, if agents of $C$ who previously submitted linear orders $R_{i_{1}}, \ldots, R_{i_{k}}$ now submit linear orders $R_{i_{1}}^{\prime}, \ldots, R_{i_{k}}^{\prime}$ while the remaining voters submit their original linear orders.

Definition 2 Let $F$ be an $S C F$ and $C=\left\{i_{1}, \ldots, i_{k}\right\} \subseteq N$ be a coalition. We say that a profile $u$ is manipulable for $F$ by the coalition $C$, if there is a profile $u$, which occurred as a result of misrepresentation by the agents of $C$ with the linear orders $R_{i_{1}}, \ldots, R_{i_{k}}$ being replaced by them with linear orders $R_{i_{1}}^{\prime}, \ldots, R_{i_{k}}^{\prime}$, if $F\left(u^{\prime}\right) R_{i_{s}} F(u)$ for all $s=1,2, \ldots, k$.

In other words, a profile is manipulable by a coalition $C$ if agents of $C$ can misrepresent their preferences in such a way that every member of $C$ will benefit from the change.

A scoring rule $F$ is characterised by the sequence of scores $v_{1} \geq v_{2} \geq \ldots \geq$ $v_{m}$. For each profile $u \in \mathcal{L}^{n}$ and for every alternative $a \in A$, we can define the score of $a$, denoted $S c_{F}(u, a)$, which can be computed as $S c_{F}(u, a)=\sum_{\ell=1}^{m} v_{\ell} i_{\ell}$, where the number $i_{k}$ shows how many times the alternative $a$ was ranked $k$ th. The alternative with the highest score is declared the winner. If more than one alternative has the greatest score, an agreed-upon tie-breaking procedure is employed to determine the winner. For most models of the behaviour of the population, the probability of a tie tends to zero as $n \rightarrow \infty$. Note that it is possible, and convenient, to assume that $v_{1}=1$ and $v_{m}=0$.

We can formalise this situation as follows. We write the linear order $R_{i}$ as

$$
a_{P_{i}(1)}>a_{P_{i}(2)}>\cdots>a_{P_{i}(m)}
$$

where $P_{i} \in \operatorname{Sym}(m)$ is a permutation from the symmetric group $\operatorname{Sym}(m)$ on $\{1,2, \ldots, m\}$. This way, we may assume that each agent $i$ chooses a permutation $P_{i} \in \operatorname{Sym}(m)$ (in effect, a permutation of the alternatives). Let $P_{i} v$ denote the result of applying the permutation $P_{i}$ to the elements of the vector $v$ in the obvious way; that is

$$
P_{i} v=\left(v_{P_{i}^{-1}(1)}, \ldots, v_{P_{i}^{-1}(m)}\right) .
$$

Then $P_{i} v$ gives us the contribution of agent $i$ to the "scoreboard" vector

$$
X_{n}=\left(S c_{F}\left(u, a_{1}\right), \ldots, S c_{F}\left(u, a_{m}\right)\right)=\sum_{i=1}^{n} P_{i} v .
$$

The winning alternative is the one corresponding to the greatest element of $X_{n}$. 
Although the score vector $v$ is not random in nature, nevertheless the following two characteristics of this vector will play a crucial role. We set

$$
\bar{v}=\frac{1}{m} \sum_{j=1}^{m} v_{j}, \text { and } \sigma_{v}^{2}=\frac{1}{m} \sum_{j=1}^{m}\left(v_{j}-\bar{v}\right)^{2} .
$$

Examples of scoring rules include the following.

Example 1 Plurality voting: $v=(1,0, \ldots, 0)$. We have $\bar{v}=1 / m$, and $\sigma_{v}^{2}=$ $(m-1) / m^{2}$.

Example 2 Borda's rule: $v=\left(1, \frac{m-2}{m-1}, \ldots, \frac{1}{m-1}, 0\right)$. We have $\bar{v}=1 / 2$, and $\sigma_{v}^{2}=$ $(m+1) / 12(m-1)$.

Example 3 Antiplurality voting: $v=(1, \ldots, 1,0)$. We have $\bar{v}=(m-1) / m$, and $\sigma_{v}^{2}=(m-1) / m^{2}$.

Example $4 k$-Approval voting: $v=(1, \ldots, 1,0, \ldots, 0)$ ( $k$ ones). We have $\bar{v}=$ $k / m$, and $\sigma_{v}^{2}=k(m-k) / m^{2}$.

Plurality and antiplurality rules are particular cases of the $k$-approval voting for $k=1$ and $k=m-1$, respectively. Brams and Sanver [7] define a fixed rule as a voting system in which voters vote for a predetermined number of candidates. Any fixed rule is a $k$-approval voting rule for some $k$. The Borda and the fixed rules will be further called classical.

The difference between $k$-approval voting and approval voting has to be emphasised. Under approval voting any voter may approve as many alternatives as she wishes, while under $k$-approval voting it is compulsory that any voter approves her exactly $k$ best alternatives. Thus approval voting is not a scoring rule. We refer the reader to the book by S. Brams and P. Fishburn [4] and to the more recent survey of the same authors [5].

\section{The Winning Margin}

This section addresses the problem: if the voters choose permutations independently and uniformly at random from $\operatorname{Sym}(m)$ (which corresponds to the IC conjecture), what is the asymptotic behaviour of the winning margin (i.e. the difference between the greatest and second-greatest elements of $X_{n}$ )? The central limit theorem is used to find the asymptotics.

\section{Proposition 1 (Central limit result.)}

$$
\frac{X_{n}-n \bar{v} 1}{\sigma_{v} \sqrt{n}} \stackrel{D}{\rightarrow} N(0, \Sigma),
$$

where $\Sigma$ is the $m \times m$ matrix with diagonal elements equal to 1 and off-diagonal elements equal to $\frac{-1}{m-1}$, and 1 is used to denote a vector of $1 \mathrm{~s}$. 
Proof The $P_{i} v$ are i.i.d. random vectors with mean $\bar{v} 1$ and covariance matrix $\sigma_{v}^{2} \Sigma$. The result follows by the classical central limit theorem (see e.g. [9], p. 170).

Define $\delta: \mathbb{R}^{m} \rightarrow[0, \infty)$ such that $\delta(x)$ is the difference between the greatest and second-greatest elements of $x$. Note that $\delta$ is continuous, with the properties $\delta(a x)=a \delta(x)$ for $a \geq 0 ; \delta(x+b 1)=\delta(x)$ for $b \in \mathbb{R}$. We can thus apply $\delta$ to both sides of (1) to obtain

$$
\frac{\delta\left(X_{n}\right)}{\sigma_{v} \sqrt{n}} \stackrel{D}{\rightarrow} \delta(Y),
$$

where $Y \sim N(0, \Sigma)$.

Proposition 2 (Convergence of expectation) We also have

$$
\frac{E\left[\delta\left(X_{n}\right)\right]}{\sigma_{v} \sqrt{n}} \rightarrow E[\delta(Y)] .
$$

Proof To establish convergence of the expectation when we already have convergence in distribution, it is sufficient to check that our family of random variables $\left\{\delta\left(X_{n}\right) / \sigma_{v} \sqrt{n}\right\}_{n=1}^{\infty}$ is bounded in mean square ([3], Corollary to Theorem 25.12). That is, $E\left[\left(\delta\left(X_{n}\right) / \sigma_{v} \sqrt{n}\right)^{2}\right]$ should be bounded, independently of $n$. For this, note that $\delta(x)=\delta(x-b 1) \leq 2\|x-b 1\|_{\infty} \leq 2\|x-b 1\|_{2}$ for all $x \in \mathbb{R}^{m}$ and $b \in \mathbb{R}$. Hence

$$
\begin{aligned}
E\left[\frac{\delta\left(X_{n}\right)^{2}}{n \sigma_{v}^{2}}\right] & \leq E\left[\frac{4\left\|X_{n}-n \bar{v} 1\right\|_{2}^{2}}{n \sigma_{v}^{2}}\right]=\frac{4}{n \sigma_{v}^{2}} \sum_{j=1}^{m} E\left[\left(\sum_{i=1}^{n}\left(v_{P_{i}^{-1}(j)}-\bar{v}\right)\right)^{2}\right] \\
& =\sum_{j=1}^{m} \sum_{i=1}^{n} E\left[\left(v_{P_{i}^{-1}(j)}-\bar{v}\right)^{2}\right]=4 m
\end{aligned}
$$

making use of the fact that for any fixed $j, v_{P_{i}^{-1}(j)}$ are independent random variables, and so the variance of their sum is the sum of their variances. The result follows.

Proposition 3 (The distribution of $\delta(Y)$ ) The random vector $Y$ has the same multivariate normal distribution as $\sqrt{\frac{m}{m-1}}(Z-\bar{Z} 1)$, where $Z=\left(Z_{1}, \ldots, Z_{m}\right) \sim$ $N\left(0, I_{m}\right)$ and $\bar{Z}=\frac{1}{m} \sum_{j=1}^{m} Z_{j}$. In particular,

$$
E[\delta(Y)]=\sqrt{\frac{m}{m-1}} E[\delta(Z)] .
$$

Proof The distribution of $Y$ is $N(0, \Sigma)$. To see that $\sqrt{\frac{m}{m-1}}(Z-\bar{Z} 1)$ also has this distribution, one only has to verify that $\operatorname{Cov}\left(Z_{j}-\bar{Z}, Z_{k}-\bar{Z}\right)=\delta_{j k}-1 / m$ and $E\left[Z_{j}-\bar{Z}\right]=0$. 
The values of $E_{m}=E[\delta(Y)]$ for various $m$ can be found from several published tables of the expected order statistics of normal variables; e.g. those in [13]. This allows the construction of the following table:

\begin{tabular}{c|ccccccccc}
$m$ & 3 & 4 & 5 & 6 & 7 & 8 & 9 & 10 & 16 \\
\hline$E_{m}$ & 1.036 & 0.845 & 0.747 & 0.685 & 0.643 & 0.611 & 0.586 & 0.567 & 0.4971
\end{tabular}

Theorem 1 The average winning margin $W M(n, m, v)=E\left[\delta\left(X_{n}\right)\right]$ is asymptotic to $E_{m} \sigma_{v} \sqrt{n}$ as $n \rightarrow \infty$.

Proof Follows from Propositions 1-3.

\section{Threshold Coalition Size}

Given a profile, in order to determine the minimum manipulating coalition size for this profile we have to know the winning margin and how the scores may change during the manipulation attempt. Suppose we are given the scoring rule with a score vector $v=\left(v_{1}, \ldots, v_{m}\right)$. Suppose also that at a certain profile the alternative $a$ wins, while $b$ has the second-best score. A voter wants to manipulate in favour of $b$. Then $b$ must have a higher rank on the ballot of this voter. The best that the voter can do is to move $b$ to the first position on her ballot and move $a$ to the last position. This will reduce the winning margin by $\left(v_{1}-v_{i}\right)+\left(v_{j}-v_{m}\right)$, where $i$ and $j$ are the original positions of $b$ and $a$ respectively; note $i<j$. The greatest amount by which any single voter will be able (and willing) to reduce the winning margin is thus

$$
d_{v}=\max _{i}\left[\left(v_{1}-v_{i}\right)+\left(v_{i+1}-v_{m}\right)\right] .
$$

For the Borda rule, $d_{v}=(m-2) /(m-1)$. For plurality and $k$-approval voting, $d_{v}=1$.

Let the threshold coalition size $M_{v}$ be (the random variable) given by

$$
M_{v}=\frac{\delta\left(X_{n}\right)}{d_{v}}
$$

where, as in the last section, $\delta\left(X_{n}\right)$ is the winning margin. Any coalition capable of manipulating the outcome of the election must have at least $\left\lceil M_{v}\right\rceil$ members. (Here $\lceil\cdot\rceil$ denotes the ceiling function: $\lceil x\rceil$ is the smallest integer not less than $x$. We will also use the floor function: $\lfloor x\rfloor$ is the greatest integer not greater than $x$.)

A note of caution must be given here. It is not true that any coalition of more than $\left\lceil M_{v}\right\rceil$ members can manipulate at the given profile. This coalition is capable of reversing the gap between the best and the second best alternative but it is possible that the third (and highly undesirable) alternative will overtake both and ruin the manipulation attempt. This can be illustrated by the following example:

Example 5 Let us consider the Borda rule $B$ and the following profile $u$ : 


\begin{tabular}{cccccc}
$R_{1}$ & $R_{2}$ & $R_{3}$ & $R_{4}$ & $R_{5}$ & $R_{6}$ \\
\hline$a$ & $a$ & $b$ & $b$ & $c$ & $c$ \\
$b$ & $b$ & $a$ & $c$ & $a$ & $b$ \\
$c$ & $c$ & $c$ & $a$ & $b$ & $a$
\end{tabular}

As $S c_{B}(u, a)=3, S c_{B}(u, b)=3.5$ and $S c_{B}(u, c)=2.5$, the winner is $b$. The coalition $\{1,2\}$ may attempt to manipulate to get $a$ chosen, submitting linear orders $R_{1}^{\prime}$ and $R_{2}^{\prime}$, where they swap the order of $b$ and $c$. By doing so, they will reverse the winning margin between $b$ and $a$ but their attempt will fail since $c$ will be chosen, which is their worst alternative.

Nevertheless $M_{v}$ is a very important parameter of the rule. We will show that for classical scoring rules this number asymptotically coincides with the minimum size of the coalition that is capable of manipulation.

Theorem 2 The average threshold coalition size, $\operatorname{ATC}(n, m, v)=E\left[M_{v}\right]$, is asymptotic to $C(m, v) \sqrt{n}$ as $n \rightarrow \infty$, where $C(m, v)=E_{m} \sigma_{v} / d_{v}$.

Proof Follows from Theorem 1 and the arguments given at the beginning of this section.

\section{Minimum Manipulating Coalition Size}

Our next goal is to prove that in a large electorate it is almost certain that a coalition will exist that can reverse the margin between the best and second-best alternatives. Moreover, for the classical voting rules it will be of size $\left\lceil M_{v}\right\rceil$ or $\left\lceil M_{v}\right\rceil+1$ (depending on the tie-breaking procedure). First, we will show that, for any constant $t$ and for any linear order $R \in \mathcal{L}$, it is almost certain that, for $n$ large enough, at least $t\left\lceil M_{v}\right\rceil$ voters will submit this linear order. To see this, note that the number of voters who submit $R$ will be of order $n$, but $\left\lceil M_{v}\right\rceil$ is of order only $\sqrt{n}$. The following result states this fact more formally.

Theorem 3 Let $R \in \mathcal{L}$ be a linear order and $u=\left(R_{1}, \ldots, R_{n}\right)$ be a random profile. Let $t>0$ be a positive number. Then, for any scoring rule with score vector $v$, there is a number $\beta>0$ such that

$$
P\left(\#\left\{i \mid R_{i}=R\right\} \geq t\left\lceil M_{v}\right\rceil\right) \geq 1-2 e^{-\beta n}
$$

for all large enough $n$. That is, the probability that a coalition of like-minded voters, who submit linear order $R$, of size $t\left\lceil M_{v}\right\rceil$ exists converges to 1 with exponential rate as $n \rightarrow \infty$.

Proof Let $D_{i}$ be the random variable which is 1 if voter $i$ submits $R$ and 0 otherwise. Let $M^{\prime}=\sum_{i=1}^{n} D_{i}$ be the number of voters who submit $R$; the claim is that $M^{\prime} \geq t M_{v}$ with high probability. Let $p=E\left[D_{i}\right]$. The IC conjecture states that $p=1 / m !>0$. Choose $\alpha \in(0, p)$; then

$$
\left(M^{\prime}<\alpha n\right) \Rightarrow\left(\frac{1}{n} \sum_{i=1}^{n}\left(D_{i}-p\right) \leq \alpha-p\right)
$$


Also,

$$
\left(M_{v}>\frac{\alpha n}{t}\right) \Rightarrow\left(\delta\left(\frac{X_{n}}{n}-\bar{v} 1\right)>\frac{\alpha d_{v}}{t}\right) \Rightarrow\left(\frac{X_{n}}{n}-\bar{v} 1 \in \delta^{-1}\left(\left[\alpha d_{v} / t, \infty\right)\right)\right) .
$$

Since $(-\infty, \alpha-p]$ and $K=\delta^{-1}\left(\left[\alpha d_{v} / t, \infty\right)\right)$ are closed sets which do not include the origin, Cramér's large-deviation theorem ([10], p.36) gives that for some $\beta>$ 0 ,

$$
P\left(\frac{1}{n} \sum_{i=1}^{n}\left(D_{i}-p\right) \leq \alpha-p\right)<e^{-\beta n} \quad \text { and } \quad P\left(\frac{X_{n}}{n}-\bar{v} 1 \in K\right)<e^{-\beta n}
$$

for all large enough $n$. It follows that

$$
P\left(M^{\prime} \geq t M_{v}\right) \geq P\left(M^{\prime} \geq \alpha n \text { and } M_{v} \leq \alpha n / t\right) \geq 1-2 e^{-\beta n}
$$

for all large enough $n$.

The following theorem was effectively proved in [15]. Here we prove a slightly stronger statement.

Theorem 4 (Kim-Roush, 1996) Any scoring voting rule $F$, with the exception of antiplurality, is coalitionally manipulable with limiting probability 1, i.e. the probability that a random profile is coalitionally manipulable for $F$ converges to 1 with exponential rate as $n \rightarrow \infty$. If $F$ is one of the classical scoring rules with the score vector $v$, then the probability of existence of manipulating coalition of size $M_{v}+1$ also converges to 1 with exponential rate.

Proof Let $u=\left(R_{1}, \ldots, R_{n}\right)$ be a random profile. Let $F(u)=w$ and $s$ is the alternative with the second-best score. Let $v$ be the score vector of $F$ and let $d=$ $v_{1}-v_{n-1}=1-v_{n-1}$. Since $F$ is different from the antiplurality, $v_{n-1} \neq 1$ and $d>0$.

Let $t>d_{v} / d$. By Theorem 3, among $R_{i}$ 's there will be at least $t\left\lceil M_{v}\right\rceil$ of linear orders $R$ for which $w$ occupies the last place and $s$ occupies second to last. Moving $s$ to the top place without changing the order of other alternatives will add $d t\left\lceil M_{v}\right\rceil$ points to the score of $s$ while the score of $w$ will remain the same and the scores of other alternatives will not increase. As $d t\left\lceil M_{v}\right\rceil>\delta\left(X_{n}\right)$, the new winner will be $s$ and this manipulation attempt will be successful.

If $F$ is classical and different from antiplurality, then $d=d_{v}$ and as we saw above $u$ will be almost surely manipulable by a coalition of size $\left\lceil M_{v}\right\rceil+1$.

The antiplurality $A$ is the exception indeed. In the three-alternative case the limiting probability of coalitional manipulability will be $1 / 2$. If alternatives are denoted $a, b, c$, then a profile with $S c_{A}(a)>S c_{A}(b)>S c_{A}(c)$ is manipulable if $S c_{A}(a)+S c_{A}(c)<2 S c_{A}(b)$ and not manipulable if $S c_{A}(a)+S c_{A}(c)>2 S c_{A}(b)$. Due to the symmetry these two events have equal probabilities. Kim and Roush [15] gave the formula for calculating the limiting probability for $m>3$.

Let $F$ be a scoring rule with a score vector $v$. Let the minimum manipulating coalition size $M C_{v}$ be (the random variable) equal to the minimum size of a coalition capable of manipulation, if the random profile is manipulable, and $\infty$ if it is 
not. Let average minimum manipulating coalition size $A M M C(n, m, v)$ be the expectancy of $M C_{v}$ conditional on the event that the random profile is manipulable.

We recap the results of this section in the following

Theorem 5 For any scoring rule different from the antiplurality,

$$
\operatorname{AMMC}(n, m, v) \geq \operatorname{ATC}(n, m, v) .
$$

The average minimum manipulating coalition size $A M M C(n, m, v)$ and average threshold coalition size $A T C(n, m, v)$, are asymptotically equal and, as $n \rightarrow \infty$, both are is asymptotic to $C(m, v) \sqrt{n}$, where $C(m, v)=E_{m} \sigma_{v} / d_{v}$.

Proof By Theorem 4 we may discard nonmanipulable profiles. Hence, the first part is clear since $M C_{v} \geq M_{v}$. For all rules but antiplurality the remainder of the theorem follows from Theorems 2 and 4 .

\section{A Drawback of the Average Minimum Manipulating Coalition Size}

We will illustrate this drawback in the case of $m=3$ alternatives, but apparently it appears for all $m \geq 3$.

Consider the scoring rule $v=(1,1-p, 0)$, where $0 \leq p \leq 1$. For a given profile, denote the winning candidate $a$, the second-placegetter $b$, and the thirdplacegetter $c$. Let $d_{1}=\delta\left(X_{n}\right)$ be the winning margin of $a$ over $b$, and $d_{2}=$ $\delta\left(-X_{n}\right)$ be the "second margin" between $b$ and $c$. Two types of manipulation in favour of $b$ are possible:

- A voter who prefers $c$ to $b$ to $a$ may mis-represent his preference as $b, c, a$. The effect of this will be to reduce the winning margin by $p$, while increasing the second margin by $2 p$.

- A voter who prefers $b$ to $a$ to $c$ may mis-represent his preference as $b, c, a$. The effect of this will be to reduce both the winning and second margins by $1-p$.

Suppose a coalition of voters contains $x_{1}$ voters of the first type and $x_{2}$ voters of the second type. Successful manipulation by this coalition requires

$$
\begin{array}{r}
d_{1}-p x_{1}-(1-p) x_{2} \leq 0, \\
d_{2}+2 p x_{1}-(1-p) x_{2} \geq 0 .
\end{array}
$$

If $0<p \leq 1 / 2$, then the smallest coalition that can succesfully manipulate has

$$
x_{1}=0, \quad x_{2}=\frac{d_{1}}{1-p}
$$

when $d_{1} \leq d_{2}$, and

$$
x_{1}=\frac{d_{1}-d_{2}}{3 p}, \quad x_{2}=\frac{2 d_{1}+d_{2}}{3(1-p)}
$$


when $d_{1} \geq d_{2}$. If, on the other hand, $1 / 2 \leq p \leq 1$, then the minimal coalition has

$$
x_{1}=\frac{d_{1}}{p}, \quad x_{2}=0 .
$$

Let us denote $x_{+}=x$, if $x \geq 0$ and $x_{+}=0$, otherwise. Then the minimum manipulating coalition size can be written as

$$
M C_{v}=\frac{1}{\max (p, 1-p)} d_{1}+\frac{1}{3}\left(\frac{1}{p}-\frac{1}{1-p}\right)_{+}\left(d_{1}-d_{2}\right)_{+}
$$

in all cases where $0<p \leq 1$. (There is a small probability that insufficiently many like-minded voters will exist to form such a minimal coalition. But by Theorem 3, this possibility can be neglected for our purposes.)

If we define $\rho: \mathbb{R}^{3} \rightarrow[0, \infty)$ by $\rho(x)=(\delta(x)-\delta(-x))_{+}$, the excess of the winning margin over the second margin, and note that for these rules $d_{v}=$ $\max (p, 1-p)$, then we can write

$$
M C_{v}=\frac{\delta\left(X_{n}\right)}{d_{v}}+\frac{1}{3}\left(\frac{1}{p}-\frac{1}{1-p}\right)_{+} \rho\left(X_{n}\right) .
$$

Note that the first term of this expression is the threshhold coalition size $M_{v}$.

The average minimum manipulating coalition size can be taken to be the expectation of this quantity. (Strictly speaking, this expectation should be conditional on manipulability of the profile. But since the probability of this event converges rapidly to 1 - by Theorem 3 again - the conditional expectation can be replaced by an unconditional one for our purposes.) Also, by the same arguments as used in section 3 , we have

$$
\frac{E\left[\delta\left(X_{n}\right)\right]}{\sigma_{v} \sqrt{n}} \rightarrow \sqrt{\frac{m}{m-1}} E[\delta(Z)] \quad \text { and } \quad \frac{E\left[\rho\left(X_{n}\right)\right]}{\sigma_{v} \sqrt{n}} \rightarrow \sqrt{\frac{m}{m-1}} E[\rho(Z)],
$$

where $Z \sim N\left(0, I_{m}\right)$. The average minimum manipulating coalition size thus satisfies

$$
\frac{A M M C(n, 3, v)}{\sigma_{v} \sqrt{n}} \approx \frac{E\left[M C_{v}\right]}{\sigma_{v} \sqrt{n}} \rightarrow \frac{E_{3}}{d_{v}}+\frac{1}{\sqrt{6}}\left(\frac{1}{p}-\frac{1}{1-p}\right)_{+} E[\rho(Z)] .
$$

When $1 / 2 \leq p \leq 1$ the second term vanishes. For such rules, the average threshhold coalition size and the average minimum manipulating coalition size have the same asymptotics. But when $0<p<1 / 2$, the value of

$$
\gamma(v)=\lim _{n \rightarrow \infty} A M M C(n, 3, v) / \sqrt{n}
$$

exceeds that of $\lim _{n \rightarrow \infty} A T C(n, 3, v) / \sqrt{n}$. For small $p$, it is much greater, and indeed $\lim _{p \rightarrow 0} \gamma(v)=\infty$.

Now let us consider the case $p=0$ : the anti-plurality rule. For this rule, only the voters of the second type have power to manipulate. If $d_{1}<d_{2}$, no manipulation is possible; otherwise the smallest manipulating coalition has

$$
x_{1}=0, \quad x_{2}=d_{1} .
$$


Hence

$$
\begin{aligned}
\operatorname{AMMC}(n, 3, v) & =E\left[M C_{v} \mid d_{1} \geq d_{2}\right] \\
& =E\left[d_{1} \mid d_{1} \geq d_{2}\right] \\
& =E\left[d_{1} 1_{d_{1} \geq d_{2}}\right] / P\left(d_{1} \geq d_{2}\right) \\
& =2 E\left[\delta\left(X_{n}\right) 1_{\delta\left(X_{n}\right) \geq \delta\left(-X_{n}\right)}\right] .
\end{aligned}
$$

(In the notation used here, $1_{Q}$ has the value 1 if the logical proposition $Q$ is true, and 0 if it is false.) So

$$
\gamma(v)=\lim _{n} \frac{A M M C(n, 3, v)}{\sqrt{n}}=2 \sigma_{v} \sqrt{3 / 2} E\left[\delta(Z) 1_{\delta(Z) \geq \delta(-Z)}\right],
$$

which is a finite number.

If we adopt the average minimum manipulating coalition size as a measure of goodness of the rule, then we will have to accept that, the closer the rule to antiplurality the better it becomes, and that there are no optimal rules. This would be clearly an absurd conclusion. Hence, no matter how attractive it may seem, the average minimum manipulating coalition size is not an appropriate measure for comparing rules. We suggest that the average threshold coalition size should be used instead.

\section{Optimal Scoring Rules}

In this section we will determine the rules optimal in relation to asymptotic average threshold coalition size. For $m=3,4$ the optimal rule is Borda Count and for $m \geq 5$ the optimal rule is $\lfloor m / 2\rfloor$-approval voting.

By Theorem 5, we may assume that the quality of the rule is determined by the ratio $\sigma_{v} / d_{v}$, or on the number $C(m, v)=E_{m} \sigma_{v} / d_{v}$ which we will analyse in this section.

Theorem 6 The asymptotic average minimum manipulating coalition size is maximised among all scoring rules by the Borda rule when the number $m$ of alternatives is 3 or 4 , and by $\lfloor m / 2\rfloor$-approval voting when $m \geq 5$.

Proof We must show that the claimed rules maximise the ratio $\sigma_{v} / d_{v}$ (or equivalently, $\sigma_{v}^{2} / d_{v}^{2}$ ) among all score vectors $v$.

Note that

$$
\sigma_{v}^{2}=\frac{1}{m} \sum_{i=1}^{m}\left(v_{i}-\bar{v}\right)^{2}=\frac{1}{2} E\left[(U-V)^{2}\right]=\frac{1}{m^{2}} \sum_{i<j}\left(v_{i}-v_{j}\right)^{2},
$$

where $U$ and $V$ are independent copies of $v_{P_{1}^{-1}(1)}$. We recall that, $v_{1}=1$ and $v_{m}=0$. If we parametrize the score vectors by setting $v_{i}=\sum_{j=i}^{m-1} t_{j}$, where $t_{1}, \ldots, t_{m-1} \geq 0$ and $\sum_{j=1}^{m-1} t_{j}=1$, then

$$
\sigma_{v}^{2}=S(t)=\frac{1}{m^{2}} \sum_{i \leq j}\left(\sum_{k=i}^{j} t_{k}\right)^{2} .
$$


Let us first consider all the score vectors $v$ with a fixed value of $d_{v}$. Among these, we only have to maximise $\sigma_{v}$. The set

$$
V_{\alpha}=\left\{\left(t_{1}, \ldots, t_{m-1}\right): \text { all } t_{i} \geq \alpha \text { and } \sum_{i=1}^{m-1} t_{i}=1\right\}
$$

includes a representation of all the score vectors with $d_{v}=1-\alpha$. Since $S$ is a convex function and $V_{\alpha}$ is a convex set, the maximum value of $S$ on $V_{\alpha}$ must be attained at an extremal point of $V_{\alpha}$, i.e. at one of the points $t^{(i)}(\alpha)$ with every component except the $i$ th equal to $\alpha$ (and the $i$ th component equal to $1-(m-2) \alpha$ ). In particular, the maximum value of $\sigma_{v}$ among all score vectors with $d_{v}=1-\alpha$ is attained by a $v$ corresponding to one of the $t^{(i)}(\alpha)$.

Now let us see that the maximum is achieved at $t^{*}(\alpha)=t^{(\lfloor m / 2\rfloor)}(\alpha)$, and also at $t^{(\lceil m / 2\rceil)}(\alpha)$. Observe that $S\left(t^{(i)}(\alpha)\right)=S\left(t^{(m-i)}(\alpha)\right)$ by symmetry, so it is enough to note that when $i<\lfloor m / 2\rfloor$,

$$
S\left(t^{(i)}(\alpha)\right)-S\left(t^{(i+1)}(\alpha)\right)<0
$$

Since $\alpha \leq \frac{1}{m-1}$, we have $1-(m-2) \alpha \geq \alpha$. Now a direct calculation shows that

$$
\begin{gathered}
S\left(t^{(i)}(\alpha)\right)-S\left(t^{(i+1)}(\alpha)\right)=\frac{1}{m^{2}}\left\{\sum_{r=1}^{i}\left((1-(m-2) \alpha+(r-1) \alpha)^{2}-(r \alpha)^{2}\right)\right. \\
\left.\quad+\sum_{r=1}^{m-i-1}\left((r \alpha)^{2}-(1-(m-2) \alpha+(r-1) \alpha)^{2}\right)\right\} \\
=\sum_{r=i+1}^{m-i-1}\left((r \alpha)^{2}-(1-(m-2) \alpha+(r-1) \alpha)^{2}\right)<0 .
\end{gathered}
$$

It now remains to find

$$
\max \left\{\frac{S\left(t^{*}(\alpha)\right)}{(1-\alpha)^{2}}: 0 \leq \alpha \leq \frac{1}{m-1}\right\} .
$$

For $m=3$, we have

$$
\frac{S\left(t^{*}(\alpha)\right)}{(1-\alpha)^{2}}=\frac{S(1-\alpha, \alpha)}{(1-\alpha)^{2}}=\frac{2\left(1-\alpha+\alpha^{2}\right)}{9(1-\alpha)^{2}}=\frac{2}{9}\left(1+\frac{\alpha}{(1-\alpha)^{2}}\right) .
$$

Since this is an increasing function of $\alpha$, its maximum is achieved when $\alpha$ takes its greatest possible value of $1 / 2$. This corresponds to the Borda rule.

For $m=4$, we have

$$
\frac{S\left(t^{*}(\alpha)\right)}{(1-\alpha)^{2}}=\frac{S(\alpha, 1-2 \alpha, \alpha)}{(1-\alpha)^{2}}=\frac{1-2 \alpha+2 \alpha^{2}}{4(1-\alpha)^{2}}=\frac{1}{4}\left(1+\frac{\alpha^{2}}{(1-\alpha)^{2}}\right) .
$$

Since this is again an increasing function of $\alpha$, its maximum is achieved when $\alpha$ takes its greatest possible value of $1 / 3$. This also corresponds to the Borda rule. 
Now we turn our attention to the case $m \geq 5$. Note that in general

$$
\begin{gathered}
S\left(t^{*}(\alpha)\right)=\frac{1}{m^{2}}\left\{\sum_{i \leq j<\lfloor m / 2\rfloor}((j-i+1) \alpha)^{2}+\sum_{\lfloor m / 2\rfloor<i \leq j}((j-i+1) \alpha)^{2}\right. \\
\left.+\sum_{i \leq\lfloor m / 2\rfloor \leq j}(1-(m-2-j+i) \alpha)^{2}\right\} \\
=c_{m}\left(1-(m-2) \alpha+\left(\frac{2+e_{m}-3 m+m^{2}}{3}\right) \alpha^{2}\right)
\end{gathered}
$$

where $c_{m}=\left(1-e_{m} / m^{2}\right) / 4$ and $e_{m}$ is 1 if $m$ is odd, 0 if $m$ is even. To complete our proof, we must show that the maximum in (5) is attained when $\alpha=0$, i.e. that $S\left(t^{*}(\alpha)\right) /(1-\alpha)^{2} \leq S\left(t^{*}(0)\right)=c_{m}$ for $0 \leq \alpha \leq 1 /(m-1)$. To this end, note that

$$
S\left(t^{*}(\alpha)\right)-c_{m}(1-\alpha)^{2}=c_{m} \alpha\left(-(m-4)+\left(\frac{-1+e_{m}-3 m+m^{2}}{3}\right) \alpha\right)
$$

Since $\frac{1}{3}\left(-1+e_{m}-3 m+m^{2}\right) \geq 0$ for $m \geq 5$, for these values of $m$ we obtain

$$
\begin{gathered}
S\left(t^{*}(\alpha)\right)-c_{m}(1-\alpha)^{2} \leq c_{m} \alpha\left(-(m-4)+\left(\frac{-1+e_{m}-3 m+m^{2}}{3}\right) \frac{1}{m-1}\right) \\
=\frac{c_{m} \alpha}{3(m-1)}\left(e_{m}-13+12 m-2 m^{2}\right) \leq 0 .
\end{gathered}
$$

This ends the proof.

The following graph and table display the numbers $C(m, v)$ for small $m$ for the plurality, Borda, $\lfloor m / 2\rfloor$-approval, and 3-approval voting. 


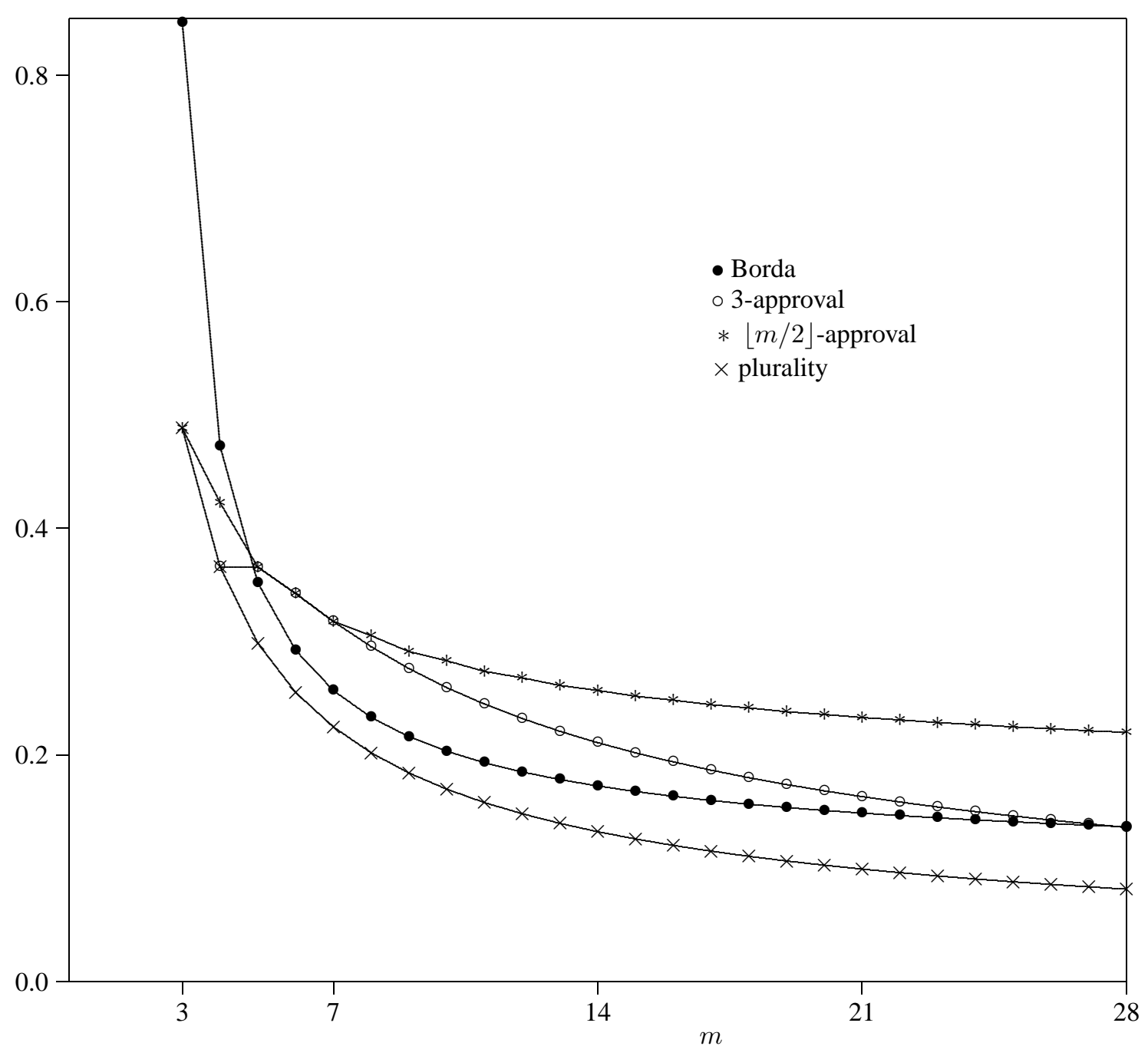

The following table gives some numerical values of the numbers $C(m, v)$ for small $m$ :

\begin{tabular}{|c|c|c|c|c|c|c|c|c|}
\hline & 3 & 4 & 5 & 6 & 7 & 8 & 9 & 10 \\
\hline Borda & 0.846 & 0.472 & 0.352 & 0.292 & 0.257 & 0.233 & 0.216 & 0.203 \\
Plurality & 0.488 & 0.366 & 0.299 & 0.255 & 0.225 & 0.202 & 0.184 & 0.170 \\
3-Appr. & & 0.366 & 0.366 & 0.343 & 0.318 & 0.296 & 0.276 & 0.260 \\
$\lfloor m / 2\rfloor$-Appr. & 0.488 & 0.423 & 0.366 & 0.343 & 0.318 & 0.306 & 0.291 & 0.284 \\
\hline
\end{tabular}

We included 3-approval voting in this table for the following reason. In the recent presidential elections in France, in which there were 16 candidates, the following experiment was conducted [16]. The experiment was run in six places: in a village, called Gy-les-Nonnains and in five voting posts in Orsay, a suburb of 
Paris. In these places voters had a possibility, after casting their official ballot, to vote unofficially according to the approval voting rule. In both places the average number of approvals on a ballot was three. So 3-approval voting may be the best approximation to approval voting among the scoring rules. Comparing it with Borda, we see that Borda eventually gets better than 3-approval but it happens only for $m \geq 28$.

This is not the first case when optimality of $\lfloor m / 2\rfloor$-approval voting manifests itself in some form. Brams and Fishburn [6] (see also [5]) proved that under approval voting the strategy of approving exactly $\lfloor m / 2\rfloor$ alternatives maximises efficacy.

\section{Conclusion}

We see that there are no optimal rules relative to the asymptotic average minimum manipulating coalition size. This characteristic is biased towards the antiplurality and should be used with great caution. The average threshold coalition size is maximised among all scoring rules by the Borda rule not only for the three-alternative case but also for the case of four alternatives. At the same time, when the number of alternatives is larger than four the $\lfloor m / 2\rfloor$-approval voting is preferable. It is interesting that in the Saari's geometric model for $m=4$ the 2-approval voting is better than Borda in respect to micro manipulation [21] and Saari expected Borda to fare poorly relative to coordinated macro manipulation. Although the difference in models should be taken into account, our results do not seem to confirm these expectations.

It is hard to imagine $\lfloor m / 2\rfloor$-approval voting implemented in practice. The best approximation to this rule would be Majoritarian Compromise which for large societies behaves as $\lfloor m / 2\rfloor$-approval voting [23].

Some experimental evidence [16] suggests that 3-approval voting might be the best approximation among scoring rules to the classical approval voting. If so, the characteristics of approval voting seem to be quite good since Borda surpasses 3 -approval only for $m \geq 28$.

It is interesting to compare these results with the results obtained by Chamberlin in [8] using computer simulation. The four rules plurality, Borda, Hare and Coombs were tested using the IC conjecture in three-alternative elections and for those profiles for which all four rules were manipulable (which is about $30 \%$ of all profiles), the average minimum manipulating coalition size was recorded in the following table.

\begin{tabular}{c|c|c} 
Voting system & 21 voters & 1000 voters \\
\hline Plurality & 2.4 & 12.4 \\
Borda & 2.3 & 15.4 \\
Hare & 1.5 & 5.9 \\
Coombs & 2.5 & 11.6
\end{tabular}

We see that for 21 voters the result of comparison is inconclusive while for 1000 voters Borda is clearly the best. 
Since for classical rules the asymptotic average minimum coalition size is the same as the asymptotic average threshold coalition size, we can compare Chamberlin's results with ours. For 1000 voters our results give us 15.4 for the plurality and 26.8 for the Borda rule. The difference is especially significant for the Borda rule. One possible explanation of this discrepancy is that in Chamberlin's simulations only profiles manipulable for all four rules, including Hare's rule, were considered. Since Hare's rule is manipulable only in $30 \%$ of all cases, this can be a rather restricted set of profiles which are more prone to manipulation than the majority of profiles.

\section{References}

1. Aleskerov F and Kurbanov E (1999) Degree of manipulability of social choice procedures. In: Current Trends in Economics. Eds: Alkan, A., Aliprantis, C.D. and Yannelis, N.C. Springer, 13-27

2. Berg S and Lepelley D (1994) On Probability models in voting theory. Statistica Neerlandica 48, 133-146

3. Billingsley P (1995) Probability and Measure 3rd ed. Wiley.

4. Brams S and Fishburn P (1982) Approval voting. Birkhauser.

5. Brams S and Fishburn P (2002) Voting Procedures. In: Handbook of Social Choice and Welfare, Volume 1, Eds: Arrow KJ, Sen A and Suzumura K, 2002, Elsevier, 173-236

6. Brams S and Fishburn P (1981) Efficacy, power and equity under approval voting. Public Choice 37: 425-434

7. Brams S and Sanver R (2003) Voter Sovereignty and Election Outcomes, to appear.

8. Chamberlin J (1985) Investigation into the relative manipulability of four voting systems. Behavior Science 30: 195-203

9. Durrett R (1996) Probability: theory and examples, 2nd ed. Duxbury Press

10. Dembo A and Zeitouni O (1993) Large deviations techniques. Jones and Bartlett

11. Favardin P Lepelley D and Serias J (2002) Borda rule, Copeland method and strategic manipulation. Review of Economic Design 7: 213 -228

12. Gibbard A (1973) Manipulation of voting schemes: A general result. Econometrica 41: 587-601

13. Harter LH (1961) Expected values of normal order statistics. Biometrika 48: 151-165

14. Kelly J (1993) Almost all social choice rules are highly manipulable, but few aren't. Social Choice and Welfare 10: 161-175

15. Kim KH and Roush FW (1996) Statistical manipulability of social choice functions. Group Decision and Negotiation 5: 263-282

16. Laslier J-P (2003) Spatial approval voting, to appear.

17. Lepelley D and Mbih B (1987) The proportion of coalitionally unstable situations under the plurality rule. Economic Letters 24, 311-315

18. Lepelley D and Mbih B (1994) The vulnerability of four social choice functions to coalitional manipulation of preferences. Social Choice and Welfare, 11: 253-265

19. Lepelley D and Valognes F (2002) Voting rules, manipulability and social homogeneity. Public Choice, forthcoming

20. Nitzan S (1985) The vulnerability of point-voting schemes to preference variation and strategic manipulation. Public Choice 47: 349-370

21. Saari D (1990) Susceptibility to manipulation. Public Choice 64:21-41

22. Satterthwaite MA (1975) Strategy-proofness and Arrow's conditions: Existence and correspondence theorems for voting procedures and social welfare functions. Journal of Economic Theory 10:187-217 
23. Slinko A (2002) The Majoritarian Compromise in Large Societies, Review of Economics Design 7(3): 343-350.

24. Smith DA (1999) Manipulability measures of common social choice functions. Social Choice and Welfare 16: 639-661 